\title{
Sustentos teóricos que fundamentan la influencia del contexto comunitario en el Desarrollo Infantil Integral de los niños de 0-5 años
}

\section{Theoretical support for the influence of the community context on the Comprehensive Child Development of children up to 5 years of age}

\author{
Martha María Fernández-Rodríguez \\ Tecnológico Universitario Espíritu Santo, Guayaquil, Ecuador \\ mmfernandez@tes.edu.ec
}

https://orcid.org/0000-0002-4765-7419

Recepción: 24/07/2021 | Aceptación: 22/10/2021 | Publicación: 30/10/2021

Cómo citar (APA, séptima edición):

Fernández-Rodríguez, M. M. (2021). Sustentos teóricos que fundamentan la influencia del contexto comunitario en el Desarrollo Infantil Integral de los niños de 0-5 años. INNOVA Research Journal, 6(3.1), 114-130. https://doi.org/10.33890/innova.v6.n3.1.2021.1842

\section{Resumen}

El presente artículo respondió a un resultado del proyecto de investigación "Influencia de la comunidad Batallón del Suburbio en el Sur de Guayaquil, en el Desarrollo Infantil Integral de los niños de 0 a 5 años", implementado por la carrera Desarrollo Infantil Integral del Tecnológico Universitario Espíritu Santo en el año 2021. Datos estadísticos de investigaciones previas realizadas por el Departamento de Estadística del Centro de Salud Santiago de Guayaquil, confirmaron la presencia de 2.021 niños de 0 a 5 años en este sector. Existiendo en dichas familias falta de preocupación por el buen desarrollo infantil integral. Todo lo cual condujo a los siguientes problemas de investigación ¿Cómo mejorar la influencia de las familias de la comunidad Batallón del Suburbio en el Desarrollo Infantil Integral de los niños de 0 a 5 años? ¿Qué sustento teórico fundamenta la influencia del contexto comunitario en el Desarrollo Infantil Integral de los niños de 0-5 años? Dicha investigación se proyectó como objetivo general "Sistematizar los sustentos teóricos que fundamentan el desarrollo Infantil Integral de los niños de 0-5 años en un contexto comunitario. La investigación fue de tipo explicativa o causal no experimental y tuvo como resultado un marco teórico de referencia para la valoración del Desarrollo Infantil Integral de los niños de 0 a 5 años de la comunidad Batallón del Suburbio. Se usaron métodos teóricos como el deductivo, analítico sintético, histórico comparativo y método de investigación cualitativa.

Palabras clave: desarrollo infantil; contexto; familia; entorno familiar. 


\begin{abstract}
This article responded to a result of the research project "Influence of the Batallón del Suburbio community in the South of Guayaquil, in the Comprehensive Child Development of children from 0 to 5 years old", implemented by the Comprehensive Child Development career of the Technological University Espiritu Santo in the year 2021. Statistical data from previous investigations carried out by the Statistics Department of the Santiago de Guayaquil Health Center, confirmed the presence of 2,021 children from 0 to 5 years old in this sector. Existing in these families lack of concern for the good integral child development. All of which led to the following research problems: How to improve the influence of the families of the Batallón del Suburbio community on the Comprehensive Child Development of children from 0 to 5 years of age? What theoretical support bases the influence of the community context on the Comprehensive Child Development of children aged 0-5? Said research was projected as a general objective "To systematize the theoretical supports that base the Comprehensive Child development of children aged 0-5 years in a community context. The research was of an explanatory or non-experimental causal type and resulted in a theoretical framework of reference for the assessment of the Comprehensive Child Development of children from 0 to 5 years of the Batallón del Suburbio community. Theoretical methods such as deductive, synthetic analytical, comparative historical and qualitative research method were used.
\end{abstract}

Keywords: child development; context; family; family environment.

\title{
Introducción
}

Lograr un Desarrollo Infantil Integral de los niños/as de 0 a 5 años debe ser una máxima aspiración de cualquier estado, el mismo debe quedar plasmado desde la Constitución como órgano superior que rige los principios básicos de la organización del Estado y los deberes y derechos ciudadanos y las normativas mínimas de la organización del gobierno, concretándose en las políticas educativas de los órganos encargados de este grupo etario. Ecuador no es la excepción y así su Carta Magna en el artículo 44 reconoce:

El Estado, la sociedad y la familia promoverán de forma prioritaria el desarrollo integral de las niñas, niños (...), y asegurarán el ejercicio pleno de sus derechos;(...). Las niñas, niños (...) tendrán derecho a su desarrollo integral, entendido como proceso de crecimiento, maduración y despliegue de su intelecto y de sus capacidades, potencialidades y aspiraciones, en un entorno familiar, escolar, social y comunitario de afectividad y seguridad. Este entorno permitirá la satisfacción de sus necesidades sociales, afectivoemocionales y culturales, con el apoyo de políticas intersectoriales nacionales y locales. (Constitución de la República del Ecuador, 2008, pág. 23)

Ello, se concreta en la Ley Orgánica de Educación Intercultural (LOEI) 2017 en su artículo 40 que define el nivel de Educación Inicial como:

Acompañamiento al desarrollo integral que considera los aspectos cognitivo, afectivo, psicomotriz, social, de identidad, autonomía y pertenencia a la comunidad y región de los niños y niñas desde los tres años hasta los cinco años de edad, garantiza y respeta sus 
derechos, diversidad cultural y lingüística, ritmo propio de crecimiento y aprendizaje, y potencia sus capacidades, habilidades y destrezas [...]. La Educación de los niños y niñas desde su nacimiento hasta los tres años de edad es responsabilidad principal de la familia, sin perjuicio de que esta decida optar por diversas modalidades debidamente certificadas por la Autoridad Educativa Nacional. (Ministerio de Educación, 2011)

Asimismo, el Código de la Niñez y la adolescencia 2003 en su artículo 37, garantiza el Derecho a la Educación que incluye en el numeral 4, el acceso efectivo a la educación inicial de cero a cinco años procurando la participación de la familia y la comunidad. (Código de la Niñéz y la Adolescencia, 2003, pág. 4)

Para alcanzar las mencionadas aspiraciones del Estado ecuatoriano, todos los organismos deben tributar a tales fines. Desde esta concepción, los niños deben gozar de un desarrollo armónico en todos los ámbitos: salud, nutricional, educativo, emocional en contextos saludables interculturales donde confluyan todos factores internos y externos en función de garantizar los derechos a un desarrollo infantil integral.

Tomando como referencia estos postulados legales y conscientes de garantizar tales derechos en "El Batallón del Suburbio", perteneciente a la Parroquia Febres Cordero en las calles "M" entre la 43 y 44 de la Ciudad de Guayaquil, Provincia del Guayas, ubicándose en la Zona 8 Distrito 4 Circuito 5; se indagó en la situación de la influencia de las familias donde investigaciones previas realizadas por el Ministerio de Salud Pública en particular por un Monitoreo 2021realizado por el Departamento de Estadística del Centro de Salud Santiago de Guayaquil, evidencian la presencia de 2.021 niños de 0 a 5 años (Ministerio, de Salud Pública, 2021). Existiendo en algunas familias ambientes socioeconómicos desfavorables predominando: falta de preocupación por el buen desarrollo infantil integral en los niños de 0 a 5 años, que los limitará en el futuro por el buen desempeño social, laboral y profesional.

En la búsqueda de alternativas de solución a las insuficiencias reflejadas en esta comunidad objeto de estudio en esta investigación, fueron consultados los aportes de autores internacionales relacionados con la influencia de los contextos comunitarios en el desarrollo infantil integral. La indagación teórica en el contexto internacional arroja estudios experimentales recientes realizados por (Fletcher, J. y Wolfe, B, 2016), quienes reseñan las consecuencias de la situación socioeconómica en el bienestar de los niños. Siguiendo la misma línea de investigación se encontraron los trabajos de (Failache, E. y Katzkowicz, N., 2019) que indagaron la relación entre nivel socioeconómico y su repercusión en el desarrollo del niño. En el contexto peruano encontramos la investigación Holgado, D. et al. (2015, pág. 103), quienes abordaron "la preparación para la prevención del trabajo infantil en tres contextos comunitarios de Jicamarca y Villa El Salvador, en el entorno periurbano de Lima (Perú), dicha investigación concreta como "el ajuste comunitario de las organizaciones educativas parece ser fundamental en la implementación efectiva de programas contra el trabajo infantil". También, se localizó la investigación desarrollada en Colombia, por Pineda-Báez, et al (2015), que evidencian los resultados de 20 años de prácticas pedagógicas de implementación del Proyecto Educativo Comunitario y reflejan como resultado: 
Sustentos teóricos que fundamentan la influencia del contexto comunitario en el Desarrollo Infantil Integral de los niños de $0-5$ años

Los saberes y las prácticas pedagógicas construidas con la primera infancia se caracterizan por ser participativas, significativas, contextuadas y humanizantes, constituyendo un tejido que se nutre además de los recursos comunitarios, del entorno y del medio ambiente, para constituirse en un proyecto de transformación social. (pág. 263)

En este ámbito colombiano, también Hernández, E., et al (2017), reflexionan "sobre el diálogo de saberes para explicar cómo este permite identificar de manera más cercana la situación, problemas y necesidades de salud que tiene la población" (pág. 242), aspectos que pueden incorporarse a la investigación que se está desarrollando en la comunidad Batallón del Suburbio, al sur de Guayaquil para valorarlos desde la perspectiva del desarrollo infantil en los niños de 0 a 5 años.

En el contexto ecuatoriano resalta la investigación de (Fiallos García, 2019, pág. 50), para quien "el apoyo social comunitario tiene estrecha relación con el apoyo social percibido, influyendo directamente en la percepción que el individuo tendrá de su comunidad y el nivel de reciprocidad que este hará a cada uno de sus miembros". Asimismo, encontramos la investigación de Moreno Acosta, César. (2016) titulada: "Análisis de la política de desarrollo infantil integral, en niñas y niños de 0 a 5 años de edad en centros infantiles del buen vivir (CIBV), quién aborda un marco teórico sobre la problemática del desarrollo infantil integral visto desde la primera infancia, su atención, los derechos de los niños y el diseño de las políticas públicas en este sentido en Ecuador, que constituyen referencias obligadas a considerar en esta investigación.

La pesquisa teórica realizada confirma la actualidad del tema y la urgencia de encontrar solución a la contradicción externa entre lo que demanda la sociedad en su Carta Magna y los diferentes entes reguladores de las políticas y derechos de los niños a las familias en cuanto a su responsabilidad por el desarrollo infantil integral y, la realidad manifiesta en dicha comunidad donde se evidencian altos índices de desnutrición, altos índices de contagios por coronavirus, condiciones de insalubridad, altos índices de ausencia de participación en programas educativos, altos índices de consumo de drogas, escaso sentido de pertenencia a una comunidad que se puede resumir en un "ambiente inadecuado, inhibidor o poco estimulante que puede repercutir de forma negativa en el desarrollo infantil" (Boato, Elvio Marcos. Henri Wallon, 2003). Todo lo cual conduce al siguiente problema de investigación ¿Cómo mejorar la influencia de las familias de la comunidad Batallón del Suburbio en el Desarrollo Infantil Integral de los niños de 0 a 5 años? Auxiliado del siguiente problema específico: ¿Qué sustento teórico fundamenta la influencia de las familias de la comunidad Batallón del Suburbio en el Desarrollo Infantil Integral de los niños de 0 a 5 años? Y tiene como objetivo general: "Sistematizar los sustentos teóricos que fundamentan el desarrollo Infantil Integral de los niños de 0-5 años en un contexto comunitario.

Tomando como punto de partida esta problemática se procede a conformar un Proyecto de Investigación que abarca a estudiantes y docentes de la carrera Desarrollo Infantil Integral del Instituto Superior Universitario Espíritu Santo, en Guayaquil. Dicho proyecto tiene como objetivo general: Contribuir al Desarrollo Infantil Integral de los niños de 0 a 5 años de la comunidad Batallón del suburbio, favoreciendo su crecimiento en contextos familiares adecuados desde un

Esta obra se comparte bajo la licencia Creative Common Atribución-No Comercial 4.0 International (CC BY-NC 4.0) Revista de la Universidad Internacional del Ecuador. URL: https://www.uide.edu.ec/ 
enfoque intercultural y contextualizado ajustados a los objetivos del Plan para Toda una Vida. Asumiendo como sustento Teórico el Paradigma sistémico en el Desarrollo Humano y familiar y la teoría bioecológica de Urie Bronfenbrenner (2001) que constituye una referencia clásica sobre el tema. Se completa los sustentos teóricos asumidos con las aportaciones de otro de los clásicas L Vigotski década del 30 con el enfoque histórico cultural y la situación social del desarrollo (SSD), quien resalta el papel del entorno y los factores internos y externos en el que se desenvuelven los niños desde los primeros años de vida que será objeto de estudio en este artículo. Coincidiendo con los criterios de quienes refieren que si "la herencia determina el potencial humano, es el medio el que intermedia las conquistas del ser humano" (Bronfenbrenner, 1996); (Gallahue, David; Ozmun, John C., 2001); (Haywood, Katheleen M.; Getchell, Nancy. , 2004).

\section{Marco Teórico}

Uno de los sustentos teóricos que se toman como referencia, es la Teoría Bioecológica (2001) de Urie Bronfenbrenner que tiene su base en el Paradigma Sistémico. Una revisión de la literatura permite ubicar las investigaciones de este autor alrededor de 14 libros y unas de 300 artículos que han constituidos sustentos sólidos para investigaciones realizadas en el ámbito de la Salud en la Enfermería, la Educación Física, la Educación la Sicología entre otras. Las investigaciones de este autor fueron evolucionando a lo largo de su vida. Ello ha llevado a críticas y reconocimientos. Según criterio de Carvalho Barreto, (2016) para no interpretar erróneamente su teoría, es necesario no fragmentar sus lecturas, sino verla como una "como teoría sistémica aplicada al estudio de la familia y el desarrollo humano". (De Carvalho Barreto, 2016, págs. 175293). Precisamente por tratarse en esta investigación de un estudio sobre la influencia de una Comunidad, en el Desarrollo Infantil Integral de los niños de 0 a 5 años, es que se toma como uno de los referentes teóricos algunos de sus postulados.

\section{Paradigma Sistémico, sus orígenes y evolución}

Se puede ubicar los orígenes del Paradigma Sistémico después de 1950 con el biólogo austriaco Ludwig Von Bertalanffy, quien consideró que "el organismo es un sistema complejo constituido por varios interconectados, que deben ser entendidos y correlacionados como una totalidad" (Vasconcelos, 2002)

Según Vasconcelos, (2002), la característica del paradigma sistémico abordado por Ludwig Von Bertalanffy, rebasa los modelos aristotélicos positivistas rompiendo con ello y, con los modelos de la Teoría de Clases, creando condiciones empíricas sólidas para producir conocimiento multidisciplinario e interdisciplinario. (Vasconcelos, 2002)

La obra de Bronfenbrenner se enmarca durante los años 70 y 80 insertándose en el paradigma sistémico en el estudio del Desarrollo Humano y familiar. Situación que, ha llevado a que muchos investigadores del tema lo ubiquen como principal líder de la Ciencia del Desarrollo. A criterio de Rogoff (2005), contribuyó a "una ciencia que salió de los laboratorios para operar la intervención y que comenzó a fundamentar otras áreas psicológicas, como la Psicología cultural" (Rogoff, 2005)

Una revisión de la evolución de la obra Teoría y Modelo Sistémico de Bronfenbrenner, permiten según criterio de Carvalho Barreto, André (2016) ubicarlo didácticamente en tres fases. 
Sustentos teóricos que fundamentan la influencia del contexto comunitario en el Desarrollo Infantil Integral de los niños de 0-5 años

Estas fases coinciden con la evolución de sus investigaciones hasta completar con lo que llamó en la tercera fase como teoría bioecológica, que constituye uno de los sustentos teóricos de esta investigación.

\section{Primera fase Juventud}

Abarca desde 1942 hasta 1970 en ella, profundiza en sus estudios sobre personalidad (Bronfenbrenner, 1960). "Este período puede considerarse como el de su transición científica de las investigaciones de la teoría de clases a la sistémica. Así se refleja en su volumen "Two Worlds of de Childhood: Us and Ussr". (De Carvalho Barreto, 2016, pág. 175).

\section{Segundo fase Vida Adulta}

Esta fase abarca desde 1971 a 1980 durante estos años consolida su modelo ecológico plasmado en el libro "La ecología del desarrollo humano: experimentos naturales y planificados" 1996, (Bronfenbrenner, 1996) donde desarrolla las principales ideas de su modelo y teoría ecológica fig. 1. Dicha teoría ecológica, está conformada por un conjunto de estructuras concéntricas en la que una está contenida dentro y a las que Bronfenbrenner, denomina microsistema, mesosistema, exosistema y macrosistema" (Escobar González, 2020, pág. 19)

\section{Figura 1}

Sistema de la Teoría Ecológica 1992 de Bronfenbrenner

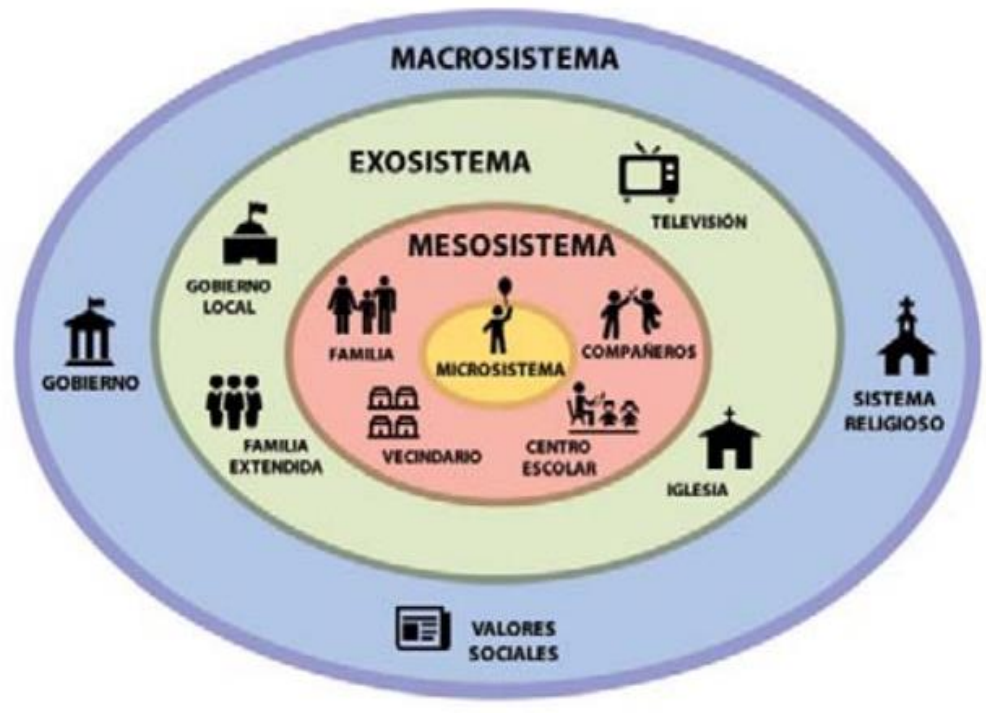

Nota: Tomado de (Escobar González, 2020, pág. 19)

\section{Tercera Fase Vejez}

Abarca 1981-2005, período en el que manifiesta su disconformidad con los aportes realizados en 1996, se aprecia una maduración de sus idas concretadas en la Teoría Bioecológica. 
Las evidencias de la misma quedan plasmadas en sus obras: "El estado de los estadounidenses: esta generación y la próxima" (Bronfenbrenner, U., McClelland, P., Wethington, E., Moen, P. y Ceci, SJ, 1996) y "Biotecnología del desarrollo humano: hacer a los seres humanos más humanos, del 2011" (Bronfenbrenner U. (., 2011), donde hace una actualización de los postulados desarrolladas a lo largo de sus investigaciones en las etapas precedentes y manifiesta concretamente las bases y evolución de su teoría sistémica.

Resumiendo, y en concordancia con (Schaffer y Kipp, 2012), "los estudios de Bronfenbrenner en la segunda y tercera fase, son una evolución de la teoría de sistema para el estudio del desarrollo familiar y humano" (Schaffer, AR y Kipp, K. , 2012). Dentro de sus postulados señala el fenómeno de continuidad y, cambio en las características biosociológicas del ser humano como individuos y grupos" (Bronfenbrenner, U., 2001, págs. 6963-6970). A decir de Carvalho Barreto, (2016) " Desde esta perspectiva, el desarrollo humano y familiar son las caras de una misma moneda, que se extiende a lo largo de la vida y el desarrollo humano, abarcando las distintas generaciones y la historia de la humanidad". (De Carvalho Barreto, 2016). A partir de esa evolución del pensamiento en el 2001, propone la teoría Bioecológica.

\section{Teoría Bioecológica y su vínculo con el Desarrollo Infantil Integral}

Siguiendo la línea de pensamiento de Bronfenbrenner, en esta investigación se aborda la Teoría Bioecológica concretada en las obras publicadas en el año 2001, "Caos Creciente en las Vidas de niños, jóvenes y familias: ¿cómo podemos revertirlo?” (Bronfenbrenner, U., 2001, págs. 197-210) y "La Teoría bioecológica del Desarrollo Humano". (Bronfenbrenner U. L.-6., 2001 a, págs. 6963-6970). En esta etapa de sus investigaciones enfatiza más en el lenguaje y los símbolos en la interacción persona contexto y enfatiza la importancia de los procesos proximales y el tiempo.

Al reformular su teoría para una comprensión bioecológica del desarrollo humano, comenzó a enfatizar, además de la interdependencia individual-contexto, las características de la persona en desarrollo en relación a las influencias de cuatro aspectos multidireccionales interrelacionados, que constituyen el modelo PPCT - persona, proceso, contexto y tiempo elementos centrales de la teoría bioecológica. ( Borges Glaus, M.A, de Souza L.R, Campos Diniz de Castro, M.A, 2015, pág. 342)

Según Martins y Szymanski (2004), citado por (Borges Glaus, M.A, de Souza L.R, Campos Diniz de Castro, M.A, 2015, pág. 342), en este modelo, persona se refiere al fenómeno de constancias y cambios a lo largo de la vida, características del individuo en desarrollo, como convicciones, nivel de actividad, temperamento, metas, motivaciones, género, entre otros. El proceso refiere respeto por la participación activa en la interacción progresivamente más complejo, recíproco con personas, objetos y símbolos en el entorno inmediato, ocurriendo regularmente y perdurable. Por contexto, se entiende el entorno en el que se inserta persona (micro, meso, exo y macrosistemas) y donde tienen lugar los procesos de desarrollo, de lo más inmediato a lo más remoto, sujeto a influencias recíprocas.

Tomando este referente en el proyecto "Influencia del contexto comunitario en el Desarrollo Infantil Integral de los niños de 0-5 años", se considerará por persona a los niños de 0 a 5 años, y la evolución en su desarrollo desde el punto de vista físico, cognitivo, psicosocial, del 
Sustentos teóricos que fundamentan la influencia del contexto comunitario en el Desarrollo Infantil Integral de los niños de 0-5 años

lenguaje y biológico nutricional, considerados aspectos internos. A referirse al proceso se aludirá a la interrelación del niño con la familia que constituye el entorno inmediato y su inserción en contextos comunitarios con influencia intercultural que favorezca su crecimiento de manera favorable influenciado por factores internos y externos como se declara en la figura 2.

Se completa los sustentos teóricos con el enfoque histórico cultural de L Vigotski en lo relativo a lo que él llama Situación Social del Desarrollo (SSD) "al principio de cada período etario se establece una relación peculiar, única e irrepetible, específica para esta etapa de la vida, entre el niño y su medio, ante todo social" (Carvajal, D., Macías, AA. y Vaca, G., 2018, pág. 1). Desde esta perspectiva, se considera factores históricos y culturales que determinan el desarrollo de la personalidad. Tomando estas consideraciones se contempla en esta investigación tener presente el desarrollo de los niños de 0 a 5 años en un contexto comunitario influenciado por los factores internos y externos, históricos y culturales. Coincidiendo con los criterios de (Mejías, 2014); (Villaman, 2016) y (Caicedo, E.; Sánchez, P; Tejeda, R., 2017), quienes afirman que SSD se debe "prestar atención a la relación entre las condiciones interpersonales o externas y las intrapersonales o internas" en la que "se definen las relaciones específicas para cada edad, exclusivas e irrepetibles entre el niño y el entorno social” (Flejalde, F, Crespo, YS. y Fernández, 2000)

Considerando que esta investigación aborda la influencia de la familia en el Desarrollo Infantil Integral y que el entorno en la que se desarrolla es una Comunidad del Batallón del Suburbio en el Sur de Guayaquil, estos postulados constituirán esencia y base para continuar el desarrollo del proyecto de investigación en dicho contexto. Ello, favorecerá un Desarrollo Infantil Integral que abarque todos los ámbitos y en contextos interculturales que le permita a futuro insertarse a la sociedad con una preparación integral.

\section{Sustento Conceptual}

Como parte de los sustentos teóricos que justifican esta investigación, se tuvieron en cuenta los conceptuales. Desde esta perspectiva fue necesario asumir que se entiende por Desarrollo Infantil Integral. En este sentido se efectúa un análisis comparativo entre el concepto Desarrollo Infantil (DI) abordado por la investigación en opción al grado científico de $\mathrm{PhD}$ "Desarrollo infantil: análisis de concepto y revisión de diagnósticos de NANDA-I" realizada por Ramallo Veríssimo de la Escuela de Enfermería de la Universidad de São Paulo, São Paulo, SP, Brasil, y el concepto presentado por el Comité Técnico Intersectorial de la Estrategia de Desarrollo Infantil Integral en Ecuador quienes lo definen como:

\section{Tabla 1}

Análisis comparativo de los Definición de Desarrollo Infantil

\begin{tabular}{|c|c|}
\hline $\begin{array}{l}\text { Definición de Desarrollo Infantil Integral } \\
\text { por el Comité Técnico Intersectorial de la } \\
\text { Estrategia de Desarrollo Integral Infantil }\end{array}$ & $\begin{array}{l}\text { Definición de Desarrollo Infantil por } \\
\text { Ramallo Veríssimo }\end{array}$ \\
\hline
\end{tabular}

Un proceso de cambios continuos por el que El Desarrollo Infantil es parte fundamental atraviesan los niños y niñas desde su del desarrollo humano, un proceso activo y 


\section{Definición de Desarrollo Infantil Integral por el Comité Técnico Intersectorial de la Estrategia de Desarrollo Integral Infantil \\ Definición de Desarrollo Infantil por Ramallo Veríssimo}

concepción que, en condiciones normales, garantizan el crecimiento, la maduración y la adquisición progresiva de las complejas funciones humanas como el habla, la escritura, el pensamiento, los afectos, la creatividad. Es un proceso multifactorial en el que influyen aspectos internos (biológicos) y externos, y en el que intervienen múltiples actores. Es por esto que el desarrollo depende de la calidad de las condiciones sociales, económicas y culturales en las que nacen, crecen y viven los niños y niñas, de las oportunidades que el entorno les ofrece y de la efectiva garantía de derechos por parte del Estado y la sociedad. (MIES-INFA., 2011)

Nota: Comparación entre las Definiciones de Desarrollo Infantil

Elaborado por la autora

\begin{abstract}
único para cada niño, expresado por la continuidad y los cambios en las habilidades motoras, cognitivas, psicosociales y del lenguaje, con adquisiciones cada vez más complejas en las funciones de la vida diaria y en el ejercicio de su rol social. El periodo prenatal y los primeros años iniciales de la infancia son cruciales en el proceso de desarrollo, lo cual constituye la interacción de las características biopsicológicas, heredadas genéticamente, con las experiencias que ofrece el entorno. El alcance del potencial de cada niño depende del cuidado que responde a sus necesidades de desarrollo. (Martins de Souza, 2015, pág. 1101)
\end{abstract}

\section{Metodología}

La metodología que se empleó en esta investigación es de tipo explicativa o causal no experimental que según Bernal (2010): "es el ideal y el culmen de la investigación no experimental, (...). En la investigación explicativa se analizan causas y efectos de la relación entre variables". (Bernal C. , 2010, pág. 122). Desde esta concepción en la presente investigación se analiza la relación entre las variables los elementos que influyen en el Contexto comunitario como factor externo y los factores que intervienen en el Desarrollo Infantil Integral como factor interno. Por ser un resultado del proyecto enmarcado en un sustento teórico se tuvo en cuenta los postulados del Paradigma sistémico en el Desarrollo Humano y familiar y la teoría bioecológica de Urie Bronfenbrenner auxiliado de las concepciones teóricas expresadas por L Vigotski en la década de los 30, y las definiciones conceptuales de Comité Técnico Intersectorial de la Estrategia de Desarrollo Integral Infantil 2011 y la señalada por Ramallo Veríssimo 2015 que serán pautas a contemplar en el análisis de la influencia de las familias de la comunidad Batallón del Suburbio en el Desarrollo Infantil Integral de los niños de 0 a 5 años. Se usaron métodos teóricos como el deductivo, analítico sintético, histórico comparativo y método de investigación cualitativa.

Se usó el método, histórico comparativo en las etapas del estudio de la obra Teoría y Modelo Sistémico de Bronfenbrenner, el deductivo que permitió tomar conclusiones generales desde los sustentos teóricos asumidos de los autores antes mencionados para obtener explicaciones particulares del Desarrollo Infantil Integral en un contexto comunitario. Para ello, se aplicó el analítico sintético en tanto se estudió el Desarrollo Infantil Integral descomponiendo los factores 
Sustentos teóricos que fundamentan la influencia del contexto comunitario en el Desarrollo Infantil Integral de los niños de 0-5 años

externos e internos que influyen en el mismo en un contexto comunitario. Asimismo, se usó el método cualitativo partiendo del criterio que este tipo de investigación según Bernal (2010) busca "entender una situación social como un todo, teniendo en cuenta sus propiedades y su dinámica" (Bernal C. , 2010, pág. 60), desde esta concepción se pretendió analizar los sustentos teóricos que fundamentan la situación social en un contexto comunitario y su influencia en el Desarrollo Infantil Integral.

El Entorno en el que se desarrolló la investigación fue en el "El Batallón del Suburbio", perteneciente a la Parroquia Febres Cordero en las calles "M" entre la 43 y 44 de la Ciudad de Guayaquil, Provincia del Guayas, ubicándose en la Zona 8 Distrito 4 Circuito 5, tomando una población de 2.021 niños de 0 a 5 años. Intervinieron como investigadores los estudiantes y docentes de la carrera Desarrollo Infantil Integral del Instituto Superior Universitario Espíritu Santo.

\section{Resultados y discusión}

La comparación entre la Definición de Desarrollo Infantil Integral por el Comité Técnico Intersectorial de la Estrategia de Desarrollo Integral Infantil (2011) y, la Definición de Desarrollo Infantil por Ramallo Veríssimo (2015), permite a esta autora realizar el siguiente análisis:

Existen aspectos comunes en ambos conceptos relacionados con:

- $\quad$ Es un proceso de cambios

- Constituye un Proceso multifactorial donde influyen aspectos internos y externos

- Influencia de condiciones económicas, sociales y culturales

- Oportunidades que el entorno le ofrece

De lo que se infiere que constituyen aspectos a tener en cuenta en la investigación objeto de este proyecto.

Sin embargo, hay otros aspectos como la efectiva garantía de los derechos por parte del estado que ofrece el concepto ecuatoriano objeto de análisis que no se contempla en el concepto que se analiza en el contexto brasileño, situación que permite a esta autora considerarlo más adecuado y asumirlo en esta investigación en tanto aborda el Desarrollo Infantil desde una concepción integral y donde el contexto y la influencia de la familia son básicos para lograrlo. Por otra parte, se considera pertinente incluir lo relacionado con "el alcance del potencial de cada niño depende del cuidado que responde a sus necesidades" (Martins de Souza, 2015, pág. 1101), que es abordado en el concepto brasileño. Ello, permite en esta investigación abordar el Desarrollo Infantil Integral (DII) asumiendo los sustentos teóricos y conceptuales declarados desde las perspectivas siguientes:

- Es un proceso de cambios

- Constituye un Proceso multifactorial donde influyen aspectos internos y externos

- Influencia de condiciones económicas, sociales y culturales

- Oportunidades que el entorno le ofrece

- Efectiva garantía de los derechos por parte del estado

- El alcance del potencial de cada niño depende del cuidado que responde a sus necesidades 
Por otra parte, se contemplan los factores internos y externos que confluyen en el Desarrollo Infantil Integral en un contexto comunitario como se reflejan en la figura 2. Los mismos serán considerados en la investigación que realiza el proyecto enfocado a mejorar la influencia de las familias de la comunidad Batallón del Suburbio en el Desarrollo Infantil Integral de los niños de 0 a 5 años.

\section{Figura 2}

Factores Internos y externos que influyen en el Desarrollo Infantil Integral en un contexto comunitario

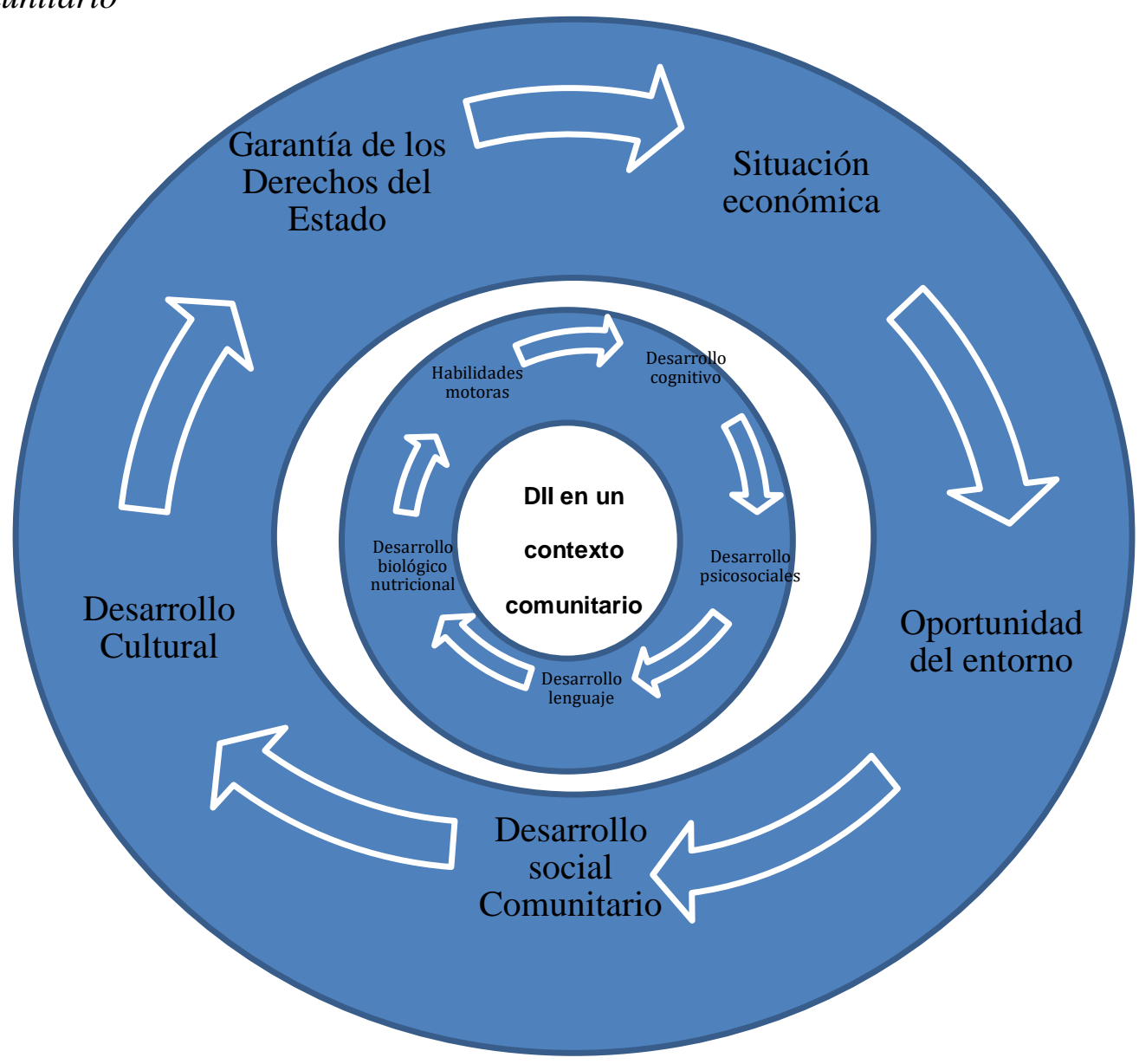

\section{Factores Externos}

- Garantía de los derechos del Estado. Ser consecuente con el cumplimiento del artículo 44 de la Constitución del Ecuador 2008 que garantiza el desarrollo integral de los niños/as y el ejercicio pleno de sus derechos, el artículo 33 en lo relativo al desarrollo intercultural, La Estrategia Nacional Intersectorial de Primera Infancia, que expresa como objetivo concretar un modelo integral e intersectorial de atención a la primera infancia con enfoque territorial, intercultural y de género. El Código de la niñez y adolescencia del 2003 en Ecuador en su artículo 37, que garantiza el acceso a la Educación Inicial, la Ley Orgánica 
Sustentos teóricos que fundamentan la influencia del contexto comunitario en el Desarrollo Infantil Integral de los niños de 0-5 años

de Educación Intercultural (LOEI) 2017 en su artículo 40 que garantiza el acompañamiento al desarrollo integral, El Reglamento General de la LOEI en su artículo 27 que determina los niveles de Educación Inicial como primer Nivel de Educación En Ecuador.

- Desarrollo cultural. Se asumirá los aspectos contemplados en la definición de Jaume Casacuberta (2007), para quien:

El desarrollo cultural comunitario contempla la cultura como eje vertebrador de procesos colaborativos, como el camino para vincular nuevos proyectos de base social, como vehículo para dinamizar, transformar y dialogar, como canalizador de iniciativas de transformación que impliquen al conjunto de una sociedad que necesita denunciar, cambiar, debatir y hacer sentir su voz a través de otros lenguajes expresivos, siempre con la finalidad de provocar cambios en positivo y de dar un papel activo y decisivo a la gente en los asuntos que conciernen a la propia comunidad. (Casacuberta, 2007). Considerando el enfoque histórico cultural de Vigostki y la SSD en la que confluyen los factores internos y externos en el desarrollo integral de los niños en un contexto comunitario será imprescindible contemplar para lograr las trasformaciones positivas de los niños.

- Desarrollo social comunitario. Está dirigido a comunidades que se encuentren en situaciones vulnerables desde el punto de vista social y cultural, estableciendo programas de desarrollo por especialistas, que impliquen cambios en los comportamientos de la población en función de su bienestar, en este caso del desarrollo infantil desde una mirada integral. "Este abordaje integral (...) implica una integralidad en la mirada, superadora de las visiones fragmentadas (...) Pero también implica una integralidad en la gestión desde la articulación intersectorial, multifactorial e interjurisdiccional." (Avalos, ML, Valladares NP; Rosero JF; Peñafiel MP; Román Z., 2018, pág. 19)

- Oportunidad del entorno. En este factor se considerará como entorno según Fernández (2010) "el conjunto de circunstancias económicas, sociales, políticas y culturales en las que nace y crece el niño en el contexto comunitario". (Fernández, 2010)

- Situación económica. Se contemplará desde la concepción de Desarrollo económico comunitario que "pueden justificar el hecho de que las iniciativas que se le asocian sean escrutadas para evaluarlo como aspecto estratégico de la intervención social" (Ninacs, 2008, pág. 101)

\section{Factores Internos}

- Desarrollo Físico: Contemplará habilidades motoras finas y gruesas. Las habilidades finas considerarán la capacidad de los niños para utilizar los músculos menores, con énfasis las manos y los dedos habilidades motoras gruesas contemplará los músculos grandes que le permitan sentarse, caminar, correr, saltar, mantener el equilibrio y cambiar de posición. Se valorará su desarrollo acorde a la edad de los niños/as. 
- Desarrollo Cognitivo: En este aspecto se considerarán las capacidades del niño relacionadas con el aprendizaje, la memoria el razonamiento, explorar su contexto con manos y ojos.

- Desarrollo Psicosocial: Partiendo que estamos en presencia de una investigación en un contexto comunitario, es básica considerar este aspecto del desarrollo del niño en el que debe formar relaciones ayudándose a sí mismo y manejar sus emociones.

- Desarrollo del Lenguaje: Este aspecto es básico en el desarrollo del niño, considera su capacidad para comprender y utilizar el lenguaje incluido el lenguaje corporal y los gestos para comunicarse. El lenguaje forma parte del Desarrollo del niño "caracterizado por la progresiva adquisición de estructuras y funciones durante las etapas tempranas de la vida, está influido por las condiciones socioeconómicas de los niños y sus familias" (Díaz AA, et al., 2017, pág. 1).

- Desarrollo biológico nutricional. Según el estado nutricional del niño será su desarrollo. Se considera el estado nutricional como:

La situación en la que el niño se encuentra en relación con la ingesta de alimentos, cómo estos son aprovechados por su organismo y son suficientes para responder al gasto dado por los requerimientos nutricionales para su edad, sexo, estado fisiológico y actividad física. Si la ingesta de alimentos es balanceada, el aprovechamiento biológico de los nutrientes será adecuado; esta relación se verá reflejada en la salud corporal del infante. (Luna J.A., Hernández I., Rojas A.F., Cadena MC, 2018, pág. 172)

Interrelacionado con los aspectos precedentes una buena nutrición en un niño permitirá un desarrollo saludable en la conducta, en lo cognitivo, en la comunicación y a futuro facilitará su rendimiento escolar. Al mismo tiempo, ayudará a adaptarse a nuevos ambientes y personas. Los niños que gozan de una buena alimentación podrán defenderse contra numerosas enfermedades infantiles que pueden de por vida dejar huellas en ellos. En criterio del Dr Reynaldo Martorell (1996), los niños desnutridos pueden verse retardados en el crecimiento y desarrollo motor, en lo cognitivo, en un bajo coeficiente intelectual, habilidades sociales deficientes, atención disminuida, problemas conductuales entre otros que afectan su desarrollo sicológico y social y cognitivo. (Martorell, 8 y 9 de abril de 1996)

Dichos aspectos se tendrán en cuenta en la investigación que se lleva a cabo sobre el Desarrollo Infantil Integral de los niños de 0 a 5 años de la comunidad Batallón del Suburbio abordándolos desde un contexto comunitario influenciado por una comunidad con particularidades históricas que hasta el momento los estudios precedentes han demostrado que no siempre, se favorece el desarrollo desde una perspectiva integral situación que limita su desarrollo a futuro.

\section{Conclusiones}

Los sustentos teóricos abordados en la Teoría Bioecológica (2001) de Urie Bronfenbrenner que tiene su base en el Paradigma Sistémico, auxiliados de las aportaciones de L Vigotski en la 
Sustentos teóricos que fundamentan la influencia del contexto comunitario en el Desarrollo Infantil Integral de los niños de $0-5$ años

década de los 30, y las definiciones conceptuales de Comité Técnico Intersectorial de la Estrategia de Desarrollo Integral Infantil 2011 y la señalada por Ramallo Veríssimo 2015, constituyeron postulados teóricos a ser considerados en la investigación "Influencia de la comunidad Batallón del Suburbio en el Sur de Guayaquil, en el Desarrollo Infantil Integral de los niños de 0 a 5 años" y permitirán el Desarrollo Infantil Integral de los niños de 0 a 5 años de esta comunidad, favoreciendo su crecimiento en contextos familiares adecuados desde un enfoque intercultural y contextualizado ajustados a los objetivos del Plan para Toda una Vida considerando el entorno y los factores internos y entornos que en influyen en el desarrollo del niño/a.

La estructura con los Factores Internos y externos desde un enfoque histórico cultural que influyen en el Desarrollo Infantil Integral en un contexto comunitario, asumida en esta investigación, constituyen puntos esenciales a indagar su comportamiento en el contexto comunitario del Batallón del Suburbio, como diagnóstico que precede la investigación sobre el Desarrollo Infantil Integral en contexto comunitario que ayudará a solventar el problema objeto de investigación en este proyecto.

\section{Limitaciones del estudio}

El hecho de asumir como uno de los sustentos teóricos los estudios de Urie Bronfenbrenner que fueron evolucionando con el de cursar del tiempo hasta el 2001, puede llevar a que sus estudios se vean de manera fragmentada lo que ha llevado a que algunos investigadores le realicen críticas y otros reconocimientos. Para que su obra no sea mal interpretada se recomienda en criterio de Carvalho Barreto, (2016) "no fragmentar sus lecturas, sino verla como una "como teoría sistémica aplicada al estudio de la familia y el desarrollo humano". (De Carvalho Barreto, 2016, págs. 175293).

Precisamente por tratarse en esta investigación de un estudio sobre la influencia de una Comunidad, en el Desarrollo Infantil Integral de los niños de 0 a 5 años, es que, se toma como uno de los referentes teóricos algunos de sus postulados, vistos en su integración de las ideas, no de manera fragmentada para evitar mal interpretaciones.

\section{Recomendaciones}

Se recomienda en investigaciones que contribuyan al desarrollo infantil integral en contextos comunitarios tener siempre presente la interrelación entre los factores internos y externos que influyen en la comunidad y que serán los principales actores que contribuirán o limitarán su desarrollo.

\section{Referencias Bibliográficas}

Avalos, ML, Valladares NP; Rosero JF; Peñafiel MP; Román Z. (2018). Los contextos familiaressocietarios para el desarrollo del aprendizaje en los niños de 0 a 5 años. Espacios, 39(20). https://www.revistaespacios.com/a18v39n20/18392019.html

Borges Glaus, M.A, de Souza L.R, Campos Diniz de Castro, M.A. (mayo-agosto de 2015). Desarrollo humano y teoría bioecológica: ensayo sobre "El contador de historias". Revista 
Quadrimestral da Associação Brasileira de Psicologia Escolar e Educacional, SP., 19(2). https://doi.org/10.1590/2175-3539/2015/0192846

Bernal, C. (2010). Metodología De La Investigación: Administración. Economía, Humanidades y Ciencias Sociales. Colombia: Pearson. https://abacoenred.com/wpcontent/uploads/2019/02/El-proyecto-de-investigaci\%C3\%B3n-F.G.-Arias-2012-pdf.pdf

Boato, Elvio Marcos. Henri Wallon. (2003). E a deficiência múltipla: uma proposta de intervenção pedagógica: Hildebrando.

Bronfenbrenner, U. (. (2011). Bioecología del desarrollo humano: hacer al ser humano más humano. A. Carvalho-Barreto (Trad.): Artmed.

Bronfenbrenner, U. (1996). La ecología del desarrollo humano: experimentos naturales y planificados: Artmed.

Bronfenbrenner, U. (2001). Caos creciente en las vidas de niños, jóvenes y familias: ¿cómo podemos revertirlo? En JC Westman (Ed.), Parenthood in America. Madison: Prensa de la Universidad de Wisconsin.

Bronfenbrenner, U. (2001). La teoría bioecológica del desarrollo humano. En NJ Smelser y PB Baltes (Eds.), Enciclopedia internacional de las ciencias sociales y del comportamiento (Vol. Vol. 10): Elsevier.

Bronfenbrenner, U. L.-6. (2001 a). a teoría bioecológica del desarrollo humano. En E. i. comportamiento. En NJ Smelser y PB Baltes (Vol. 10): Elsevier.

Bronfenbrenner, U., McClelland, P., Wethington, E., Moen, P. y Ceci, SJ. (1996). El estado de los estadounidenses: esta generación y la próxima. (Free Press. Cairns, RB, Elder, GH y Costello, EJ (Organizaciones), (1996). ed.): Cambridge University Press.

Caicedo, E.; Sánchez, P; Tejeda, R. (2017). Reflexiones en torno a las competencias profesionales. Revista Electrónica Formación y Calidad Educativa, 5(2). http://refcale.uleam.edu.ec/index.php/refcale/article/view/1761

Carvajal, D., Macías, AA. y Vaca, G. (2018). Consideraciones acerca de la teoría de la Situación Social del Desarrollo y la concepción de la Complejidad. Revista Cubana de Educación Superior, 37(3). https://bit.ly/3GymX6W

Casacuberta, J. (2007). Desarrollo cultural comunitario, presentación en sociedad. Revista de Educación Social (7). https://eduso.net/res/revista/7/enfoques/desarrollo-culturalcomunitario-presentacion-en-sociedad

Código de la Niñez y la Adolescencia. (2003). Ley No. 2002-100. https://bit.ly/3pMQLa2

Constitución de la República del Ecuador. (2008). Quito. https://bit.ly/3w0I7Wj

De Carvalho Barreto, A. (2016). Paradigma Sistémico en el Desarrollo Humano y Familiar: La Teoría Bioecológica de Urie Bronfenbrenner. Psychology in Review, 22(2). http://pepsic.bvsalud.org/scielo.php?script=sci_abstract\&pid=S1677-

$11682016000200003 \& \operatorname{lng}=\mathrm{es} \& n r m=\mathrm{iso} \& \operatorname{lng}=\mathrm{es}$

Díaz AA, Bacallao Gallestey J, Vargas-Machuca R, Aguilar Velarde R. Desarrollo infantil en zonas pobres de Perú. Rev Panam Salud Publica. 2017;41:e71. https://www.scielosp.org/article/rpsp/2017.v41/e71/

Escobar González, E. (2020). Aproximaciones conceptuales de la Teoría Ecológica para la convivencia escolar: un plan de acción. Sinopsis Educativa Revista Venezolana de 
Sustentos teóricos que fundamentan la influencia del contexto comunitario en el Desarrollo Infantil Integral de los niños de $0-5$ años

Investigación, $20(2)$.

http://revistas.upel.edu.ve/index.php/sinopsis_educativa/article/view/8358/4972\#

Failache, E. y Katzkowicz, N. (2019). Desarrollo infantil en Uruguay: una aproximación a sus determinantes. Revista desarrollo y sociedad, 83(55-104). https://doi.org/10.13043/dys.83.2

Fernández, M. (2010). El Trabajo Político - Ideológico En La Secundaria Básica Holguinera, a través de la enseñanza de la Historia Local. [Tesis en opción al grado científico de Doctor en Ciencias Pedagógicas. Cuba: Universidad de Ciencias Pedagógicas José de la Luz y Caballero.]

Fiallos García, G. A. (2019). Percepción del apoyo social comunitario en familias nucleares del CAMI pascuales [tesis de pregrado]. Universidad de Guayaquil, Guayaquil, Ecuador. https://eduso.net/res/revista/7/enfoques/desarrollo-cultural-comunitario-presentacionen-sociedad

Flejalde, F, Crespo, YS. y Fernández. (2000). El enfoque humanista del desarrollo: posible desde L.S. Vigotsky. Revista Cubana de Psicología. http://pepsic.bvsalud.org/pdf/rcp/v17n1/04.pdf

Fletcher, J. y Wolfe, B. (abril de 2016). La importancia de la renta familiar en la formación y evolución de habilidades no cognitivas en la infancia. Economics of Education Review, 54. https://doi.org/10.3386/w22168

Gallahue, David; Ozmun, John C. (2001). Compreendendo o desenvolvimento motor: bebês, crianças, adolescentes e adultos: Phorte.

Haywood, Katheleen M.; Getchell, Nancy. (2004). Desenvolvimento motor ao longo da vida: 3. ed. Artmed.

Hernández, E., Lamus, F., Carratalá, C., y Orozco, D. (2017). Diálogo de saberes: propuesta para identificar, comprender y abordar temas críticos de la salud de la población. Revista Salud Uninorte, 33(2), 242-251. http://www.scielo.org.co/scielo.php?pid=S0120$55522017000200242 \&$ script $=$ sci_abstract\&tlng=en

Holgado, D., Santolaya, F, Maya, I., Cueto, R. Rogger y Anaya, R. (2015). Preparación comunitaria y organizativa contra el trabajo infantil en tres colegios de barrios periféricos de Lima (Perú). Revista Apuntes de Psicología, 33(3),103-116. http://apuntesdepsicologia.es/index.php/revista/article/view/571

Luna J.A., Hernández I., Rojas A.F., Cadena MC. (2018). Estado nutricional y neurodesarrollo en la primera infancia. Rev Cubana Salud Pública. 44(4),172 https://www.scielosp.org/pdf/rcsp/2018.v44n4/169-185/es

Martins de Souza, J. y. (noviembre- diciembre de 2015). Desarrollo infantil: análisis de un nuevo concepto. Rev. Latino-Am. Enfermagem, 23(6). https://doi.org/10.1590/01041169.0462 .2654

Martorell, R. (8 y 9 de abril de 1996). "Undernutrition During Pregnancy and Early Childhood and its Consequences for Behavioral Development." Ponencia preparada para la conferencia del Banco Mundial sobre el Desarrollo Infantil Temprano: Invirtiendo en el Futuro (Early Chil. https://journals.sagepub.com/doi/pdf/10.1177/156482659902000304

Mejías, J. (2014). Acercamiento al enfoque histórico cultural en el contexto de la Psicología en Colombia, Pereira. http://repositorio.utp.edu.co/dspace/handle/11059/4912 
MIES-INFA. (2011). Desarrollo Infantil Integral; Conceptualización: Sistema de Formación Tejiendo el Buen Vivir.

Ministerio de Educación. (2011). Ley Orgánica de Educación Intercultural (LOEI).

Ministerio de Salud Pública. (2021). Monitoreo 2021-realizado por el Departamento de Estadística del Centro de Salud Santiago de Guayaquil.

Moreno Acosta, César. (2016). Análisis de la política de desarrollo infantil integral, en niñas y niños de 0 a 3 años de edad, en centros infantiles del buen vivir (CIBV). [Tesis (Maestría en Gerencia para el Desarrollo. Mención en Gerencia Social Universidad Andina Simón Bolívar, Sede Ecuador. Área de Gestión]. https://repositorio.uasb.edu.ec/handle/10644/5403

Ninacs, W. (2008). Desarrollo económico comunitario como estrategia para enfrentar la pobreza. Trabajo Social, 10. https://revistas.unal.edu.co/index.php/tsocial/article/view/14087/14848

Noguieras, L. M. (1996). La práctica y la teoría del desarrollo comunitario. Descripción de un modelo: Narcea.

Pineda-Báez, N. Y., Garzón-Rodríguez, J. C., Bejarano-Novoa, D. C. \& Buitrago Rodríguez, N. E. (2015). Aportes para la educación inicial: saberes construidos por el Proyecto Pedagógico Educativo Comunitario. Revista Latinoamericana de Ciencias Sociales, Niñez y Juventud, 13 (1), pp. 263-278. https://doi.org/10.11600/1692715x.13115050914

Rogoff, B. (2005). La naturaleza cultural del desarrollo humano: Artmed.

Schaffer, AR y Kipp, K. (2012). Psicología del desarrollo: infancia y adolescencia.: Cengage.

Vasconcelos, M. (2002). Pensamiento sistémico: el nuevo paradigma de la ciencia. Campinas: Papirus. ISBN-10 8530806816

Villaman, M. (2016). Aproximaciones conceptuales desde la escuela Vigotskiana a la teoría de las representaciones sociales. Psicologia em Estudo, 21(1). 